\title{
Динамика абсолютно прозрачных каналов рассеяния электронов в трехбарьерных структурах при двухфотонных переходах
}

\author{
(C) А.Б. Пашковский \\ $\mathrm{AO}$ „НПП «ИСТОК» им. Шокина“, \\ 141195 Фрязино, Московская область, Россия \\ E-mail: solidstate10@mail.ru
}

(Получена 14 июня 2016 г. Принята к печати 20 июня 2016 г.)

Для несимметричных трехбарьерных резонансно-туннельных структур с тонкими высокими барьерами решение уравнения Шредингера, описывающее резонансные переходы между тремя квантовыми уровнями в сильном двухчастотном электрическом поле, обобщено на случай отстройки частот и энергий от строго резонансных. Исследована зависимость формы резонансных уровней от амплитуды и частоты электрических полей. Показано, что в зависимости от амплитуды СВЧ поля число областей абсолютной прозрачности может доходить до четырех.

DOI: 10.21883/FTP.2017.04.44335.8171

\section{1. Введение}

Один из наиболее интересных физических эффектов в многобарьерных квантовых структурах с приложенным резонансным высокочастотным электрическим полем появление абсолютно прозрачных каналов рассеяния. С момента появления первых работ [1,2], посвященных этому вопросу, он продолжает привлекать пристальное внимание теоретиков [3-5]. В большинстве таких исследований рассматриваются переходы между двумя уровнями с итоговым испусканием или поглощением одного фотона. Естественно, при резонансном воздействии сильного одночастотного поля на двухуровневую систему происходит многократное последовательное поглощение и излучение фотонов внешнего поля, причем поглощение сменяется излучением, излучение снова поглощением и т.д. [6]. Так как конечным результатом процесса является переход электрона между уровнями с испусканием или поглощением одного фотона, далее для удобства имеет смысл называть такие переходы однофотонными.

Однако, как оказалось, абсолютно прозрачные каналы рассеяния в многобарьерных структурах могут появляться и при многофотонных переходах (в том смысле, что, проходя через структуру, электрон испускает два или более фотонов) [7-9]. Более того, было показано [9], что в трехбарьерных структурах с общим для двух ям средним квантовым уровнем в условиях когерентного транспорта электронов независимо от параметров структуры для любой амплитуды сильного резонансного поля в первой яме существует такая амплитуда резонансного поля во второй яме, что структура становится абсолютно прозрачной и большинство электронов (в пределе все), падающих на верхний резонансный уровень, могут отдавать два фотона разной частоты и уходить из структуры по нижнему уровню без промежуточного взаимодействия с фононами. Было также показано [10], что при однофотонных переходах при отстройке частоты высокочастотного поля и энергии электронов от резонансной поведение квантовых уровней может быть весь- ма нетривиальным (интересная физическая трактовка этим явлениям дана в работе [5]). Кроме теоретического интереса существует сугубо практический вопрос: если использовать подобную структуры в качестве активной ячейки квантовых каскадных лазеров с баллистическим транспортом электронов [11] (по оценкам [12] они могут быть намного эффективнее приборов, в которых рассеяние играет важную роль [13-17]), то как точно должен быть настроен резонатор и насколько может отличаться энергия электронов от строго резонансной, для того чтобы такая структура еще продолжала достаточно эффективно функционировать и преобразовывать энергию электронов в СВЧ колебания.

Поэтому представляет интерес исследовать поведение двухфотонных переходов при изменении ширины квантовых уровней, амплитуды поля и смещении частоты относительно резонансной.

\section{2. Результаты расчетов}

Как и в работе [9], рассмотрим несимметричную трехбарьерную структуру с тонкими ( $\delta$-образными) барьерами (рис. 1), к которой приложено однородное высокочастотное электрическое поле

$$
\begin{aligned}
E(t) & =2 E_{1} \cos \left(\omega_{10}+\Delta \omega_{1}\right) t+2 E_{2} \cos \left[\left(\omega_{20}+\Delta \omega_{2}\right) t+\varphi\right] \\
& =E_{1}\left(e^{i \omega_{1} t}+e^{-i \omega_{1} t}\right)+E_{2}\left(e^{i \omega_{2} t+i \varphi}+e^{-i \omega_{2} t-i \varphi}\right) .
\end{aligned}
$$

Моноэнергетический поток электронов с энергией $\varepsilon+\delta \varepsilon$ падает слева на резонансный уровень с номером $K$ первой двухбарьерной структуры (центр уровня соответствует энергии $\varepsilon$ ), частота $\omega_{10}=\omega_{1}-\Delta \omega_{1}$ соответствует переходам из центра уровня $K$ в центр уровня $L$ этой же структуры, один из резонансных уровней второй двухбарьерной структуры с номером $N$ находится вблизи уровня $L$ первой, образуя общий расщепленный (двукратно вырожденный) резонансный уровень всей структуры, а во второй яме еще ниже находится уровень с номером $I$. Частота $\omega_{20}$ соответствует 


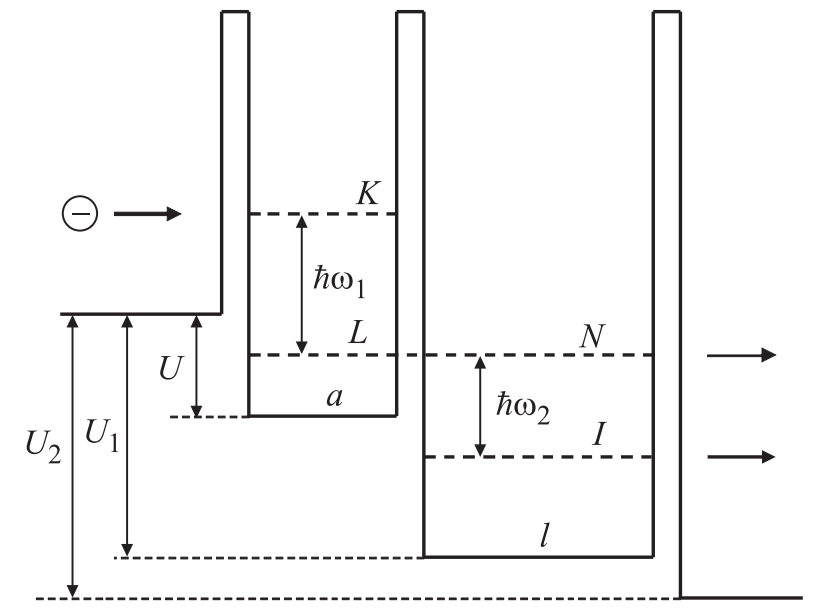

Pис. 1. Схематическая зонная диаграмма рассматриваемой трехбарьерной структуры.

переходам с уровня $N$ на уровень $I$. Соответственно здесь $\Delta \omega_{1}$ и $\Delta \omega_{2}$ - отстройки частот от строго резонансных. Следует отметить, что, вообще говоря, все переходы совершаются в непрерывном спектре, т.е. не между уровнями, а между квазиуровнями: узкими областями энергий, где внутри структуры волновая функция электронов имеет резонансный вид. В случае туннельнопрозрачных структур эти квазиуровни соответствуют максимумам коэффициента прохождения. В рассматриваемом случае условия на уровень определяются из требований максимума амплитуды волновой функции внутри структуры.

В переходах участвует только один из расщепленных уровней [9], так как второй отстоит от него хотя и на малое, но обычно заметно превышающее ширину уровня расстояние [18]. Поэтому далее во избежание путаницы для простоты будем говорить только об одном среднем уровне $L N$, на который совершаются переходы.

Возможны две принципиально отличающиеся друг от друга ситуации: узкие резонансные уровни, когда время жизни электронов на уровне заметно больше характерных времен рассеяния, и именно эти процессы определяют поведение электронов, и достаточно широкие уровни, на которых время жизни существенно меньше характерных времен рассеяния, а значит, транспорт через них можно считать баллистическим, и в этом случае все определяется только взаимодействием с высокочастотным электрическим полем. Современные технологии давно позволяют создавать барьеры столь малой толщины (из 1-2 атомных слоев) [19,20], что в гетероструктурах на основе арсенида галлия при характерных размерах квантовых ям в несколько нанометров и расстояниях между уровнями в десятки мэВ (соответствующие частоты переходов от единиц до десятков ТГц) ширина уровня может достигать несколько мэВ. В то же время по самым жестким оценкам (по подвижности электронов в полях $\sim 1 \mathrm{\kappa B} / \mathrm{cM}$, а не по их низкополевой подвижности, которая заметно больше) для объемного материала при температуре жидкого азота время жизни на уровне шириной 1 мэВ $\left(\tau \approx 0.6 \cdot 10^{-12} \mathrm{c}\right)$ уже меньше характерного времени рассеяния по импульсу $\left(\tau_{p} \approx 0.9 \cdot 10^{-12} \mathrm{c}\right)$. В то же время, как косвенно свидетельствуют экспериментальные данные [21], наличие размерного квантования существенно (как минимум в 1.5 раза) увеличивает характерные времена рассеяния. Таким образом, при температуре жидкого азота начиная с ширин уровней $>2$ мэВ характерные времена жизни на уровнях становятся как минимум в 5 раз меньше характерных времен рассеяния даже для гетероструктур на основе арсенида галлия. Использование более узкозонных материалов делает требования на ширины уровней и температуры структур еще менее жесткими. Следовательно, электронный транспорт через резонансные структуры может быть баллистическим в достаточно широком интервале изменения толщин барьеров. Далее рассматривается именно такая ситуация. Поэтому рассеянием внутри структуры, а соответственно и переходами между расщепленными уровнями пренебрегается. Пренебрегается также и неравновесными эффектами в контактных областях [22,23]: исследование их влияния на электронный транспорт несомненно важная, но совершенно другая задача.

Возможны две существенно различающиеся ситуации: расстояние между уровнями одинаково,

$$
\hbar\left|\omega_{1}-\omega_{2}\right|<\Gamma<\Sigma ;
$$

расстояние между уровнями существенно различается,

$$
\hbar\left|\omega_{1}-\omega_{2}\right| \gg \Gamma \text {. }
$$

Здесь $\Sigma$ - расстояние между расщепленными уровнями, $\Gamma$ - максимальная из ширин уровней, $\omega_{1}$ и $\omega_{2}-$ частоты резонансных переходов в первой и второй ямах соответственно. В этом случае переходы в каждой яме определяются только полем соответствующей частоты. Именно этот случай и будет рассмотрен далее. Естественно, при равенстве резонансных частот и соблюдении условия (2) все полученные результаты будут применимы и для первого случая.

Нестационарное уравнение Шредингера для такой системы имеет вид

$$
\begin{gathered}
i \hbar \frac{\partial \psi}{\partial t}=-\frac{\hbar^{2}}{2 m^{*}} \frac{\partial^{2} \psi}{\partial x^{2}}+H(x) \psi+H(x, t) \psi, \\
H(x)=U[\theta(x)-\theta(x-a)]+U_{1}[\theta(x-a)-\theta(x-a-l)] \\
+U_{2} \theta(x-a-l)+\alpha \delta(x)+\alpha \rho \delta(x-a)+\alpha \gamma \delta(x-a-l), \\
H(x, t)=-q\left[E_{1}\left(e^{i \omega_{1} t}+e^{-i \omega_{1} t}\right)+E_{2}\left(e^{i \omega_{2} t+i \varphi}+e^{-i \omega_{2} t-i \varphi}\right)\right] \\
\times\{x[\theta(x)-\theta(x-a-l)]+(a+l) \theta(x-a-l)\} .
\end{gathered}
$$

Здесь $q, m^{*}$ - заряд и масса электрона, $\alpha=\varphi_{b} b-$ мощность первого барьера, $\varphi_{b}, b$ - его высота и ширина, $\theta(x)$ - единичная ступенчатая функция Хэвисайда, $\gamma, \rho$ - численные коэффициенты, $a, l-$ расстояния между барьерами, $U, U_{1}$ и $U_{2}$ - величины скачков дна зоны проводимости на барьерах. 
Далее структура расчетов во многом аналогична используемой в работе [9], однако во избежание недоразумений часть выкладок имеет смысл воспроизвести. Решение задачи ищется по теории возмущений. Известно [3,12], что при переходах в одной яме с верхнего резонансного уровня на нижний компонента волновой функции, соответствующая поглощению фотонов и переходам вверх (не на резонансный уровень), мала. Важно отметить, что это верно только при переходах между резонансными уровнями в одной яме (прямые переходы). При переходах между резонансными уровнями в соседних ямах (диагональные переходы) вклад от переходов вверх (на виртуальный уровень) и обратно может быть не мал [24]. То же самое верно и для переходов с нижнего резонансного уровня на верхний. Поэтому, так как в данной задаче рассматриваются именно прямые переходы (см. рис. 1), волновая функция электронов имеет вид

$$
\begin{gathered}
\psi(x, t)=\psi_{K}(x) e^{-i \omega_{0} t}+\psi_{L N}(x) e^{-i\left(\omega_{0}-\omega_{1}\right) t} \\
+\psi_{I}(x) e^{-i\left(\omega_{0}-\omega_{1}-\omega_{2}\right) t}
\end{gathered}
$$

Как было показано ранее [3,12], для таких структур (далее для рассматриваемой структуры все сделанные предположения также будут проверены непосредственными расчетами) при переходах между резонансными уровнями и вблизи них волновая функция электронов во всех порядках теории возмущений с точностью до малой величины порядка $k / y$ не меняет свой вид. Поэтому можно записать

$$
\begin{aligned}
& \psi_{K}(x)= \\
& = \begin{cases}\exp \left(i k_{0} x\right)+D \exp \left(-i k_{0} x\right), & x<0, \\
A \sin (k x)+B \cos (k x), & 0<x<a, \\
Z \sin \left[k_{1}(x-a)\right]+W \cos \left[k_{1}(x-a)\right], & a<x<a+l, \\
C \exp \left[i k_{2}(x-a-l)\right], & x>a+l,\end{cases} \\
& \psi_{L N}(x)=F_{1} \\
& \times \begin{cases}D_{-} \exp (\kappa x), & x<0, \\
A_{-} \sin \left(k_{-} x\right)+B_{-} \cos \left(k_{-} x\right), & 0<x<a, \\
Z_{-} \sin \left[k_{1-}(x-a)\right]+ & \\
\quad+W_{-} \cos \left[k_{1-}(x-a)\right], & a<x<a+l, \\
C_{-} \exp \left[i k_{2-}(x-a-l)\right], & x>a+l,\end{cases} \\
& \times \begin{cases}D_{--} \exp \left[\kappa_{1}(x-a)\right], & 0<x<a, \\
Z_{--} \sin \left[k_{1--}(x-a)\right]+ & \\
+W_{--} \cos \left[k_{1--}(x-a)\right], & a<x<a+l, \\
C_{--} \exp \left[i k_{2--}(x-a-l)\right], & x>a+l .\end{cases}
\end{aligned}
$$

При достаточно мощных барьерах (а только при таких барьерах вообще и имеет смысл говорить о системе с резонансными уровнями) в отсутствие переменного электрического поля при прохождении строго через центр резонансного уровня коэффициенты волновой функции (6) равны:

$$
\begin{gathered}
F_{1}=F_{2}=0, D=D^{r}=1, B=B^{r}=1, A=A^{r}=2 y / k \\
W=W^{r}=\frac{2(-1)^{K}}{\rho}, \quad Z=Z^{r}=\frac{2 \operatorname{ctg}\left(k_{1} l\right)}{\rho} \\
C=C^{r}=-\frac{2 k_{1}}{\gamma \rho y \sin k_{1} l} .
\end{gathered}
$$

Здесь и далее $k_{i}$ - соответствующие волновые векторы на каждом уровне в соответствующей области пространства, $y=2 m^{*} \alpha / \hbar^{2}-$ величина, имеющая размерность волнового вектора, которая в структурах с $\delta$-образными барьерами выступает в роли резонансного параметра [25]. При достаточно мощных барьерах и соответственно узких резонансных уровнях $y \gg k$ и разложение решений ведется по большому безразмерному параметру $y / k$, соответственно коэффициент $A$ (коэффициент при резонансной части волновой функции) велик, а коэффициент $C$ мал (структура в отсутствие высокочастотного поля практически непрозрачна для падающих слева электронов).

Коэффициенты (7) получаются из условий сшивания волновой функции (6) на барьерах в отсутствие высокочастотного поля, которые приводят к соответствующей системе линейных уравнений $[3,9,25]$. В условиях резонанса определитель этой системы $\Delta_{K}^{r}$ мал по большому параметру $y$. При отклонении энергии влетающих электронов от резонансной на величину $\delta \varepsilon$ определитель данной системы (аналогично [12]) можно представить в виде

$$
\Delta_{K}=\Delta_{K}^{r}\left(1+\frac{i \delta \varepsilon}{\Gamma_{K}}\right)
$$

где $\Gamma_{K}-$ полуширина уровня $K$. В то же время остальные определители при решении системы методом Крамера меняются мало. Поэтому при отклонении энергии влетающих электронов от резонансной на величину $\delta \varepsilon$ внутри структуры и справа от нее в отсутствие высокочастотного поля волновая функция электронов принимает вид

$$
\psi_{K}=\frac{\psi_{K}^{r}}{1+i \delta \varepsilon / \Gamma_{K}} .
$$

Общий резонансный уровень $L N$ задается условиями [9]

$$
\cos k_{-} a \approx(-1)^{L}, \cos k_{1-} l \approx(-1)^{N},
$$$$
\sin k_{-} a \approx-\frac{k_{-}}{y}\left(\beta+\frac{\Delta \beta}{y}\right), \sin k_{1-} l \approx-\frac{k_{1-}}{y}\left(\xi+\frac{\Delta \xi}{y}\right) \text {, }
$$

$$
\beta=\frac{1+\rho+\gamma+\gamma \xi+(-1)^{L+1} \gamma \xi \rho}{\gamma+\rho+(-1)^{L+1} \gamma \xi \rho}(-1)^{L},
$$$$
\Delta \beta=(-1)^{L+1} \kappa-\frac{\gamma^{2} \Delta \xi}{\left[\gamma+\rho+(-1)^{L+1} \gamma \xi \rho\right]^{2}} .
$$

В первом порядке теории возмущений при частоте, соответствующей переходам на нижний резонансный уровень, поправка к волновой функции, соответствующая поглощению фотонов, мала $[3,12]$, а система уравнений для определения коэффициентов $A_{-}, B_{-}, C_{-}, D_{-}, Z_{-}$, 
$W_{-}$, получаемая из условий сшивания волновой функции на барьерах $[3,9]$, в матричной форме имеет вид

$$
\begin{gathered}
\left(\begin{array}{cccccc}
1 & 0 & -1 & 0 & 0 & 0 \\
i k_{0-}-y & k_{-} & 0 & 0 & 0 & 0 \\
0 & \sin k_{-} a & \cos k_{-} a & 0 & -1 & 0 \\
0 & -k_{-} \cos k_{-} a & k_{-} \sin k_{-} a & k_{1-} & -\rho y & 0 \\
0 & 0 & 0 & \sin k_{1-} a & \cos k_{1-} a & -1 \\
0 & 0 & 0 & -k_{1-} \cos k_{-} a & k_{-} \sin k_{1-} a & i k_{2-}-y
\end{array}\right) \times\left(\begin{array}{l}
D_{-} \\
A_{-} \\
B_{-} \\
Z_{-} \\
W_{-} \\
C_{-}
\end{array}\right)=\left(\begin{array}{l}
f_{1} \\
f_{2} \\
f_{3} \\
f_{4} \\
f_{5} \\
f_{6}
\end{array}\right) \\
f_{1}=\varphi_{-}(0), f_{2}=-\varphi_{-}(0), \quad f_{3}=\chi_{-}(a)-\varphi_{-}(a), \\
f_{4}=\rho y \chi_{-}(a)-\chi_{-}^{\prime}(a)+\varphi_{-}(a), \quad f_{5}=P_{-}-\chi_{-}(a+l), \quad f_{6}=\left(y-i k_{1}\right) P_{-}-\chi_{-}^{\prime}(a+l) .
\end{gathered}
$$

Здесь $\varphi_{ \pm}, \chi_{ \pm}$- частные решения уравнения (4) для $\psi_{-}$ (см. $[3,9])$ в соответствующих областях.

$$
\begin{gathered}
P_{ \pm}=\mp \frac{q E a}{\hbar \omega} \psi_{0}(a+l), \\
\varphi_{ \pm}, \chi_{ \pm}=\mp \frac{q E a}{\hbar \omega} \psi_{0}(x)+\frac{q E}{m^{*} \omega^{2}} \psi_{0}^{\prime}(x) .
\end{gathered}
$$

При энергии электронов на уровне $L N$, строго соответствующей резонансу, определитель этой системы $\Delta_{L N}^{r}$ мал по большому параметру $y$. При отклонении энергии на величину $\delta \varepsilon$ и отстройке частоты на величину $\Delta \omega_{1}$ определитель системы можно представить в виде

$$
\Delta_{L N}=\Delta_{L N}^{r}\left[1+\frac{i\left(\delta \varepsilon-\hbar \Delta \omega_{1}\right)}{\Gamma_{L N}}\right],
$$

где $\Gamma_{L N}$ - полуширина уровня $L N$.

Поэтому при отклонении энергии влетающих электронов от резонансной на величину $\delta \varepsilon$ и отстройке частоты от резонансной на $\Delta \omega_{1}$ волновая функция на среднем уровне в первом порядке теории возмущений $\left(F_{1}=1\right.$, $\left.F_{2}=0\right)$ принимает вид

$$
\psi_{L N}=\frac{\psi_{L N}^{r}}{\left(1+i \delta \varepsilon / \Gamma_{K}\right)\left[1+i\left(\delta \varepsilon-\hbar \Delta \omega_{1}\right) / \Gamma_{L N}\right]} .
$$

В условиях строгого резонанса $\left(\delta \varepsilon=\Delta \omega_{1}=0\right)$, коэффициенты волновой функции на среднем уровне, рассчитанные в первом порядке теории возмущений $\left(F_{1}=1\right)$, равны [9]

$$
\begin{gathered}
C_{-}=-\frac{4 i q E_{1} y^{2}\left[\gamma+\rho+(-1)^{L+1} \gamma \xi \rho\right]}{m^{*} \omega_{1}^{2} k_{2-}}=Z_{-} \frac{k_{1-}}{\gamma y}=\frac{W_{-}}{1-\gamma \xi}, \\
D_{-}=-\frac{4 i q E_{1} y^{2}\left[\gamma+\rho+(-1)^{L+1} \gamma \xi \rho\right]^{2}}{m^{*} \omega_{1}^{2} k_{2-}}=B_{-}=A_{-} \frac{k_{-}}{y},
\end{gathered}
$$

а коэффициенты волновой функции на нижнем уровне, рассчитанные во втором порядке теории возмущений $\left(F_{1}=F_{2}=1\right)$, есть

$$
\begin{aligned}
D_{--} & =W_{--}=\gamma C_{--}=Z_{--} \frac{k_{1--}}{y} \\
& =\frac{8 q E_{1} E_{2} \gamma^{3}\left[\gamma+\rho+(-1)^{L+1} \gamma \xi \rho\right] y^{4}}{\left(m^{*}\right)^{2} \omega_{1}^{2} \omega_{2}^{2} k_{2-} k_{2--}} e^{i \varphi} .
\end{aligned}
$$

Аналогично случаям верхнего и среднего уровней определитель системы линейных уравнений для получения коэффициентов волновой функции на нижнем уровне из условия непрерывности волновой функции на границах областей при отстройке от строгого резонанса на энергию $\delta \varepsilon$ и отстройке частот от строго резонансных на величины $\Delta \omega_{1}, \Delta \omega_{2}$ можно представить в виде

$$
\Delta_{I}=\Delta_{I}^{r}\left[1+\frac{i\left[\delta \varepsilon-\hbar\left(\Delta \omega_{1}+\Delta \omega_{2}\right)\right]}{\Gamma_{I}}\right],
$$

где $\Gamma_{I}-$ полуширина уровня $I$. Поэтому при отклонении энергии влетающих электронов от резонансной на величину $\delta \varepsilon$ и отстройке частот от резонансной на $\Delta \omega_{1}, \Delta \omega_{2}$ волновая функция на нижнем уровне во втором порядке теории возмущений $\left(F_{1}=1, F_{2}=1\right)$ принимает вид

$$
\begin{aligned}
\psi_{I}=\psi_{I}^{r} /[1 & \left.+\frac{i \delta \varepsilon}{\Gamma_{K}}\right]\left[1+\frac{i\left(\delta \varepsilon-\hbar \Delta \omega_{1}\right)}{\Gamma_{L N}}\right] \\
& \times\left[1+\frac{i\left[\delta \varepsilon-\hbar\left(\Delta \omega_{1}+\Delta \omega_{2}\right)\right]}{\Gamma_{I}}\right] .
\end{aligned}
$$

Здесь $\psi_{I}^{r}-$ волновая функция на нижнем уровне в условиях строгого резонанса $\left(\delta \varepsilon=\Delta \omega_{1}=\Delta \omega_{2}=0\right)$.

Рассчитав поправку второго порядка к волновой функции основного состояния, можно получить, что для верхнего уровня внутри структуры и за ней, как и предполагалось ранее, с точностью до малых величин порядка $k / y$

$$
\psi_{2}(x)=-z \psi_{0}(x)
$$

где

$$
\begin{gathered}
z=z_{0} \frac{1}{1+i \delta \varepsilon / \Gamma_{K}} \frac{1}{1+i\left(\delta \varepsilon-\hbar \Delta \omega_{1}\right) / \Gamma_{L N}}, \\
z_{0}=\left(\frac{2 q E_{1}}{m^{*} \omega_{1}^{2}}\right)^{2} \frac{\left[\gamma+\rho+(-1)^{L+1} \gamma \xi \rho\right]^{2} y^{4}}{k_{0} k_{2-}} .
\end{gathered}
$$

Аналогично в третьем порядке теории возмущений можно рассчитать поправку к волновой функции электронов на среднем резонансном уровне:

$$
\psi_{L N}(x) \approx \psi_{-}(x)-(z+w) \psi_{-}(x) .
$$


Здесь коэффициент $z$ отвечает за вклад верхнего резонансного уровня, коэффициент $w-$ нижнего,

$$
\begin{aligned}
& w= w_{0} \frac{1}{1+i\left(\delta \varepsilon-\hbar \Delta \omega_{1}\right) / \Gamma_{L N}} \\
& \times \frac{1}{1+i\left[\delta \varepsilon-\hbar\left(\Delta \omega_{1}+\Delta \omega_{2}\right)\right] / \Gamma_{I}} \\
& w_{0}=\left(\frac{2 q E_{2}}{m^{*} \omega_{2}^{2}}\right)^{2} \frac{y^{4} \gamma^{4}}{k_{2-} k_{2--}} .
\end{aligned}
$$

Как и раньше, форма волновой функции с точностью до малых величин порядка $k / y$ не изменяется. Так как при расчете поправок второго и третьего порядков вид волновой функции не меняется, то аналогично [9] процедуру расчета поправок более высоких порядков можно продолжить и получить коэффициенты волновой функции на каждом резонансном уровне в виде постоянных множителей и знакопеременных рядов:

$$
\begin{aligned}
1-(z & +w)+(z+w)^{2}-(z+w)^{3}+\ldots \\
& +(-1)^{n+1}(z+w)^{n} \ldots=1 /(1+z+w)
\end{aligned}
$$

для среднего и нижнего резонансного уровней и

$$
\begin{aligned}
& 1-z+z(z+w)-z(z+w)^{2}+\ldots \\
& \quad+(-1)^{n} z(z+w)^{n} \ldots=(1+w) /(1+z+w)
\end{aligned}
$$

для верхнего. Таким образом, в сильном поле коэффициенты волновой функции электронов (6) принимают вид

$$
\begin{gathered}
D=B_{0} \frac{1+w}{1+w+z}-1, \quad A=A_{0} \frac{1+w}{1+w e+z}, \\
B=B_{0} \frac{1+w}{1+w+z}, \quad Z=Z_{0} \frac{1+w}{1+w+z}, \\
W=W_{0} \frac{1+w}{1+w+z}, \quad C=C_{0} \frac{1+w}{1+w+z}, \\
F_{1}=F_{2}=\frac{1}{1+w+z}, \quad B_{0}=\frac{B^{r}}{1+i \delta \varepsilon / \Gamma_{K}}=\frac{2}{1+i \delta \varepsilon / \Gamma_{K}} \\
A_{0}=\frac{A^{r}}{1+i \delta \varepsilon / \Gamma_{K}}, \quad Z_{0}=\frac{Z^{r}}{1+i \delta \varepsilon / \Gamma_{K}}, \\
W_{0}=\frac{W^{r}}{1+i \delta \varepsilon / \Gamma_{K}}, \quad C_{0}=\frac{C^{r}}{1+i \delta \varepsilon / \Gamma_{K}}
\end{gathered}
$$

отношение потока электронов, уходящих по нижнему уровню, к потоку электронов, уходящих по среднему, есть

$$
\frac{j_{--}}{j_{-}}=\frac{k_{2--}\left|C_{--}\right|^{2}}{k_{2-}\left|C_{-}\right|^{2}}=w_{0} \frac{\Gamma_{I}^{2}}{\Gamma_{I}^{2}+\left[\delta \varepsilon-\hbar\left(\Delta \omega_{1}+\Delta \omega_{2}\right)\right]^{2}}=\xi,
$$

a для коэффициентов отражения от рассматриваемой трехбарьерной структуры $(R)$ и прохождения по среднему $\left(T_{-}\right)$и нижнему $\left(T_{--}\right)$уровням получаем

$$
R=\left|\frac{B_{0}(1+w)}{1+z+w}-1\right|^{2}, T_{-}=\frac{1-R}{1+\xi}, T_{--}=\xi \frac{1-R}{1+\xi} .
$$

Как отмечалось выше, при данном подходе учитываются все переходы, при которых электрон многократно последовательно испускает и поглощает фотоны разной частоты, однако для удобства и наглядности переходы с уходом электронов из системы по среднему уровню будем называть однофотонными, переходы с уходом электрона по нижнему уровню - двухфотонными.

Автоматически для данной задачи получается и критерий на сильное поле (слабыми можно считать поля, когда $\left.z_{0}, w_{0} \ll 1\right)$. Ранее [11] было показано, что параметр $z_{0}$ принимает значение порядка единицы (поле становится сильным, и свойства структуры могут сильно меняться), если амплитуда СВЧ поля имеет напряженность, при которой в статическом поле той же напряженности на расстояниях, равных ширине ямы, электрон набирает энергию порядка ширины уровня (в нашем случае, с учетом требований на баллистический транспорт электронов, $\sim 1$ мэВ на расстояниях $\sim 100 \AA$, соответствующая напряженность электрического поля $\sim 1 \mathrm{kB} / \mathrm{cm})$. Следует отметить, что СВЧ поле, которое для данной задачи считается сильным, на самом деле достаточно слабое (а иногда и очень слабое) поле для большинства полупроводниковых приборов. Необходимо сделать еще одно замечание: радиусы сходимости рядов (23) и (24) равны 1. Однако ранее [26] для двухбарьерных структур было показано, что подобные решения можно далеко (по крайне мере до $|z| \approx(y / k)^{2}$ ) продолжить за радиус сходимости соответствующих рядов. Так как решения данных задач во многом аналогичны, можно ожидать, что это справедливо и в рассматриваемом случае.

Выражение для коэффициента отражения после соответствующей подстановки носит простой, но довольно громоздкий вид и неудобно для аналитических исследований, однако оно сильно упрощается в случае полного прохождения через центр верхнего уровня $z_{0}=w_{0}+1$ [9] при строго резонансных частотах $\Delta \omega_{1}=\Delta \omega_{2}=0$ :

$$
\begin{aligned}
R= & -4 z_{0} \Gamma_{K}^{2} \Gamma_{L N}^{2}\left(z_{0} \Gamma_{I}^{2}+\delta \varepsilon^{2}\right) /\left\{z _ { 0 } ^ { 2 } \Gamma _ { L N } ^ { 2 } \left[4 \Gamma_{K}^{2} \Gamma_{I}^{2}\right.\right. \\
& \left.+\left(\Gamma_{K}+\Gamma_{I}\right)^{2} \delta \varepsilon^{2}\right]+\left(\Gamma_{K}^{2}+\delta \varepsilon^{2}\right)\left[\left(\Gamma_{L N}+\Gamma_{I}\right)^{2}+\delta \varepsilon^{2}\right] \delta \varepsilon^{2} \\
& -2 z_{0} \Gamma_{K} \Gamma_{L N}\left[\Gamma_{I}^{2}-\Gamma_{K} \Gamma_{L N}+\Gamma_{I}\left(\Gamma_{K}+\Gamma_{L N}\right)\right] \delta \varepsilon^{2} \\
& \left.-2 z_{0} \Gamma_{L N}\left(\Gamma_{I}+\Gamma_{K}\right) \delta \varepsilon^{4}\right\}
\end{aligned}
$$

Коэффициент прохождения через структуру в этом случае $(T=1-R)$ при различных амплитудах СВЧ поля в зависимости от расстояния до центра уровня приведен на рис. 2. Здесь и далее величины приведены в безразмерных единицах, однако, как отмечалось ранее, требования на баллистический транспорт электронов накладывают определенные ограничения на ширины уровней, а соответственно и на характерные частоты. Поэтому следует помнить, что, например, для структур на основе GaAs при температуре жидкого азота соответствующие 


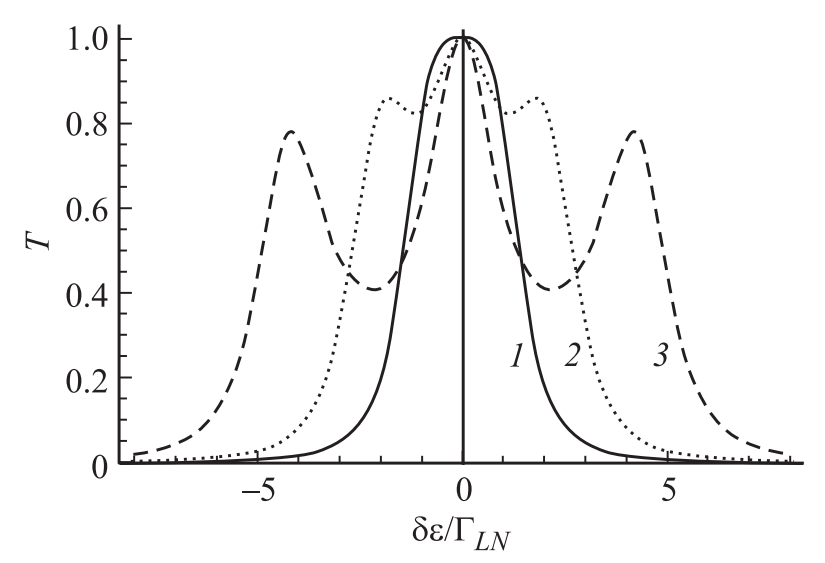

Pис. 2. Зависимость коэффициента прохождения $T$ от расстояния до центра уровней $\Gamma_{K}=\Gamma_{L N}=\Gamma_{I}, z_{0}=1$ (1), 3 (2), 10 (3).

полуширины уровней Г должны быть $\sim 1$ мэВ и больше, соответствующие этой энергии сдвиги частот -240 ГГц и более $(\hbar \Delta \omega \approx \Gamma)$, сами частоты переходов соответственно составляют от единиц до десятков ТГц.

Видно, что с ростом амплитуды поля центральный уровень вначале заметно расширяется, а затем от него

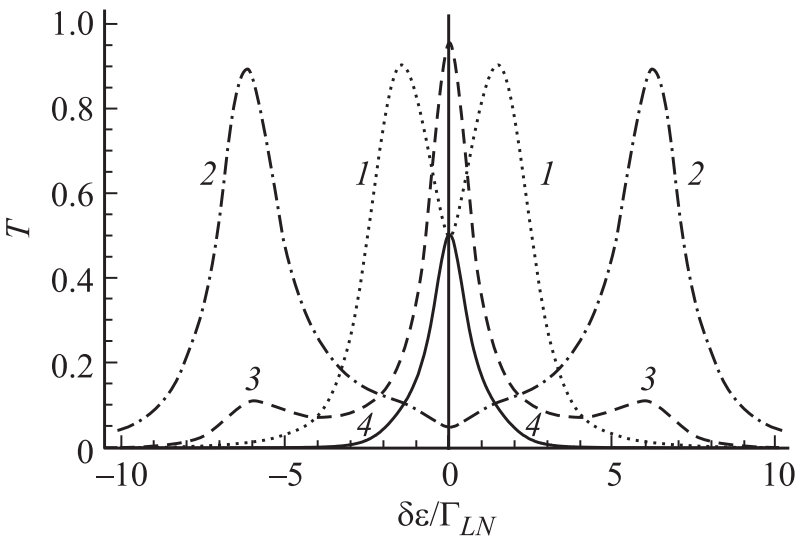

Рис. 3. Зависимости коэффициента прохождения $T$ по среднему уровню $z_{0}=2(1), 20$ (2) и по нижнему уровню $z_{0}=2$ (3), 20 (4) от расстояния до центра уровней $\Gamma_{K}=1.5 \Gamma_{L N}, \Gamma_{I}=0.5 \Gamma_{L}$

как бы отщепляются еще два уровня одинаковой ширины. Это отщепление можно назвать условным, так как в минимуме коэффициент прохождения оказывается достаточно велик. Таким образом, прозрачность структуры с ростом амплитуды СВЧ поля существенно возрастает. Можно показать, что при условии $\Gamma_{K}=\Gamma_{L N}+\Gamma_{I}$ в
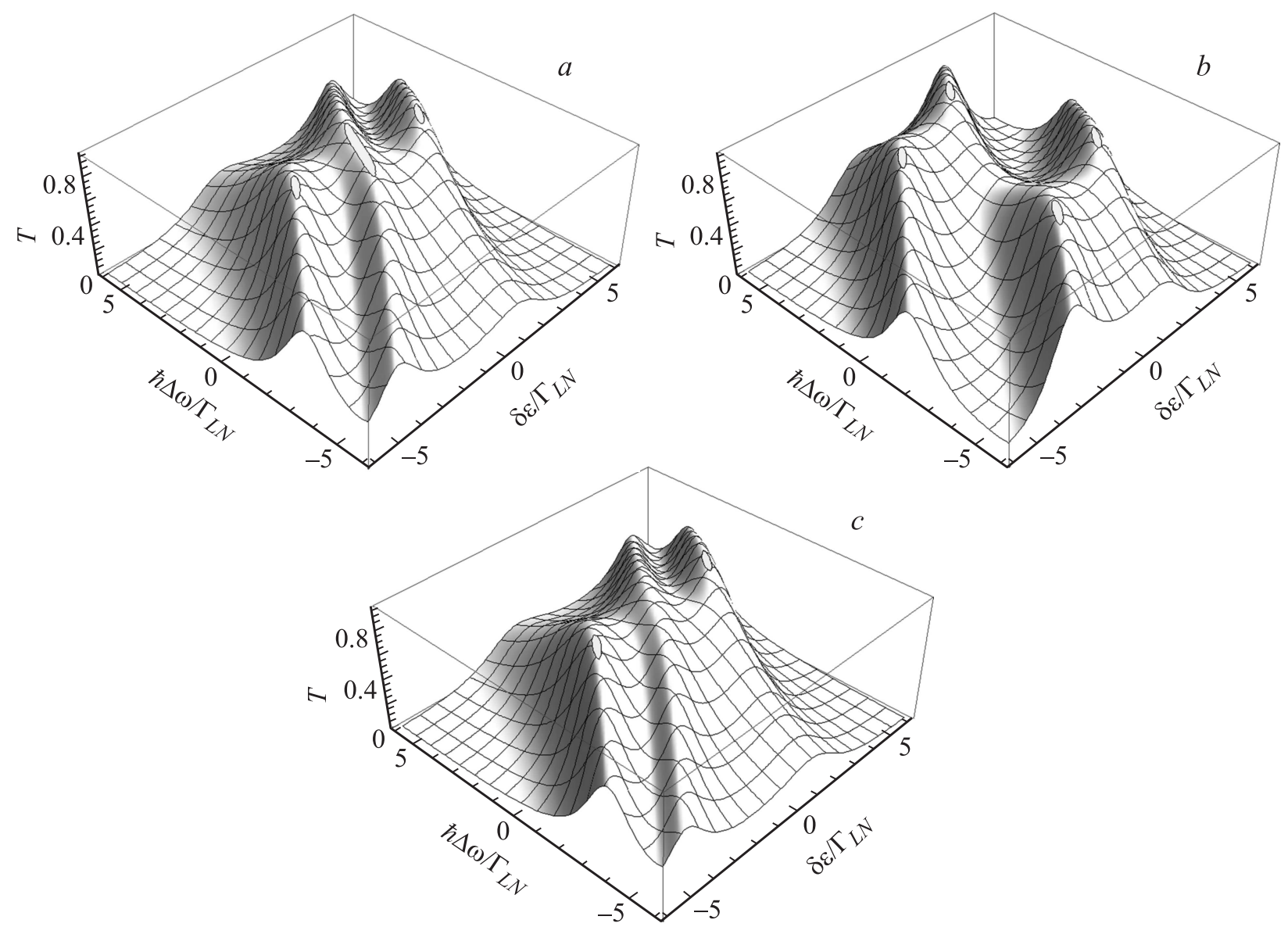

Рис. 4. Зависимости коэффициента прохождения $T$ электронов через трехбарьерную структуру от расстояния до центра уровней и отклонения частоты от резонансной $\Gamma_{K}=2 \Gamma_{L N}, \Gamma_{L}=\Gamma_{I}, z_{0}=4, w_{0}=3(a), 11(b), 2(c)$. Овалами выделены области с $T>99 \%$. 
максимуме каждого пика при

$$
z_{0}=\frac{\left(\Gamma_{I}+\Gamma_{L N}\right)^{2}+\delta \varepsilon^{2}}{\Gamma_{L N}\left(2 \Gamma_{I}+\Gamma_{L N}\right)}
$$

мы будем наблюдать строго единичный коэффициент прохождения (рис. 3). Естественно, единицу дает сумма потоков по нижнему и центральному уровням.

При этом определяющее значение имеет отношение ширин среднего и нижнего уровней: если ширина нижнего намного больше ширины среднего, большинство электронов уходят из структуры, отдав два фотона; если ширины сравнимы, то в центральном максимуме коэффициента прохождения с ростом поля электроны уходят в основном по нижнему уровню [9], а в боковых максимумах в основном по центральному уровню (рис. 3).

В случае, когда ширина нижнего уровня много меньше ширины среднего $\left(\Gamma_{I} \ll 0.5 \Gamma_{L N}\right)$, средний максимум просто сильно сужается (в соответствии с шириной $\Gamma_{I}$ ), а коэффициенты прохождения по боковым максимумам через центральный уровень становятся практически равными 1. По сути дела в этом случае в пределе мы просто получаем двухуровневую систему с уровнями одинаковой ширины [10].

Не менее интересно поведение коэффициента прохождения и в общем случае, при произвольной отстройке частоты от резонансной. На рис. 4 приводится коэффициент прохождения при различных соотношениях нормированных амплитуд СВЧ поля в первой и второй ямах. Овалами выделены области, в которой коэффициент отражения $<1 \%$, т. е. по сути дела области абсолютной прозрачности. Видно, что в случае абсолютного прохождения в центре уровня $\left(w_{0}=z_{0}-1\right)$ наблюдаются три области абсолютной прозрачности (рис. 4,a). С ростом амплитуды поля во второй яме средний уровень разделяется на два и появляются четыре области абсолютной прозрачности (рис. 4,b), а с уменьшением - область абсолютной прозрачности в центре исчезает и остаются только два пика по краям (рис. 4,c). Естественно, особенности динамики каналов высокой прозрачности существенно зависят от соотношений между ширинами уровней. Однако даже приведенные результаты позволяют сделать вывод о том, что данная структура может быть эффективной рабочей ячейкой квантового каскадного лазера с баллистическим транспортом электронов, не требующей сверхточной настройки по частоте и энергии.

\section{3. Заключение}

Для несимметричных трехбарьерных резонанснотуннельных структур с тонкими высокими барьерами получены простые выражения для коэффициента прохождения через структуру с учетом конечной ширины резонансных уровней и исследована форма резонансных уровней от амплитуды и частоты сильных высокочастотных резонансных полей. Найдены условия, при которых на точных резонансных частотах один абсолютно прозрачный уровень расщепляется на три абсолютно прозрачных. Показано, что в условиях, когда частоты полей не являются строго резонансными, в зависимости от амплитуды СВЧ поля число областей абсолютной прозрачности может доходить до четырех. Таким образом показано, что при баллистическом транспорте электронов через трехбарьерные структуры с тонкими высокими барьерами и приложенными высокочастотными полями, близкими к резонансным, образование абсолютно прозрачных каналов рассеяния - по сути дела стандартное явление.

\section{Список литературы}

[1] M. Butiker, R. Landauer. Phys. Rev. Lett., 49 (23), 1739 (1982).

[2] М.Ю. Сумецкий, М.Л. Фельштын. Письма ЖЭТФ, 53 (1), 24 (1991).

[3] Е.И. Голант, А.Б. Пашковский. Письма ЖЭТФ, 63 (7), 559 (1996).

[4] В.Ф. Елесин. ЖЭТФ, 112 (8), 483 (1997).

[5] Н.В. Ткач, Ю.А. Сети. Письма ЖЭТФ, 95 (5), 296 (2012).

[6] Н.Б. Делоне, В.П. Крайнов. Атом в сильном световом поле (М., Энергоатомиздат, 1984) с. 7.

[7] А.Б. Пашковский. Письма ЖЭТФ, 89 (1), 32 (2009).

[8] В.Ф. Елесин, Ю.В. Копаев. ЖЭТФ, 123 (6), 1308 (2003).

[9] А.Б. Пашковский. ФТП, 45 (6), 759 (2011).

[10] А.Б. Пашковский. Письма ЖЭТФ, 93 (10), 620 (2011).

[11] Е.И. Голант, А.Б. Пашковский, А.С. Тагер. Письма ЖТФ, 20 (21), 74 (1994).

[12] Е.И. Голант, А.Б. Пашковский. ЖЭТФ, 112 (7), 237 (1997).

[13] А.Ф. Казаринов, Р.А. Сурис. ФТП, 6, 135 (1972).

[14] J. Faist, F. Capasso, D.L. Sivco. Science, 264, 553 (1994).

[15] S. Blaser, M. Rochat, M. Beck, J. Faist. Phys. Rev. B, 61 (12), 8369 (2000).

[16] C. Gmachl, F. Capasso, D.L. Sivco, A.Y. Cho. Rep. Prog. Phys., 64, 1533 (2001),

[17] M.A. Belkin, F. Capasso, F. Xie, A. Belyanin, M. Fischer, A. Wittmann, J. Faist. Appl. Phys. Lett., 92, 201101 (2008).

[18] А.Б. Пашковский. Электрон. техн. Сер. 1, СВЧ-техника, 492 (4), 17 (2007).

[19] T.C.L.G. Sollner, D.W. Goodhue, P.E. Tahnenwald, C.D. Parker, D.D. Peck. Appl. Phys. Lett., $43(6), 588$ (1985).

[20] E.R. Brown, T.C.L.G. Sollner, C.D. Parker, D.W. Goodhue, C.L. Chen. Appl. Phys. Lett., 55 (17), 1777 (1989).

[21] В.М. Лукашин, А.Б. Пашковский, К.С. Журавлев, А.И. Торопов, В.Г. Лапин, Е.И. Голант, А.А. Капралова. ФТП, 48, 684 (2014).

[22] А.Б. Пашковский. Письма ЖЭТФ, 73 (11), 698 (2001).

[23] И.А. Обухов. Неравновесные эфбекты в электронных nриборах (М., Вебер, 2010) c. 104.

[24] И.И. Абрамов. Нано- и микросистемная техника, 109 (8), 7 (2009).

[25] В.М. Галицкий, Б.М. Карнаков, В.И. Коган. Задачи по квантовой механике (М., Наука, 1981) с. 150.

[26] Е.И. Голант, А.Б. Пашковский. Теоретическая и математическая физика, 120 (2), 332 (1999).

Редактор Л.В. Шаронова 
Full transparency two photon scattering channel dynamics in triple-barrier structures

A.B. Pashkovskii

JOINT-STOCK COMPANY „Research \& Production

Corporation" «ISTOK»named after A.I. Shokin"

141190 Fryazino, Moscow reg., Russia

Abstract For asymmetric triple-barrier resonant-tunneling structures with thin and high $(\delta)$ barriers, Schrödinger equation solution that describes resonant transitions, which happen in the large-signal high-frequency electric fields taking into account levels width and frequency shift has been obtained. The levels width dependence on amplitude of intensive high frequency electric field frequencies have been investigated. It is shown, that depending on high frequency electric field amplitude number of full transparency regions can reach four. 